\title{
N-Substituted amino acids as multifunctional additives used in cooling water. Part II: N-carboxymethyl and phosphonomethyl amino acids
}

\author{
J. Telegdi ${ }^{1,2}$ \\ ${ }^{l}$ Óbuda University, Faculty of Light Industry and Environmental Engineering, \\ Doberdó u. 6., 1034 Budapest, Hungary \\ ${ }^{2}$ Department of Interfaces and Surface Modification, Institute of Materials and \\ Environmental Chemistry, Research Centre for Natural Sciences, Hungarian Academy \\ of Sciences, Magyar tudósok körútja 2, 1117 Budapest, Hungary \\ E-mail: telegdi.judit@ttk.mta.hu
}

\begin{abstract}
The aim of research was to find additives with different capacities that can control the undesired corrosion and scale formation at the same time. Amino acids with different (carboxymethyl and/or phosphonomethyl) substituents were investigated to prove their multifunctional effectiveness. The most interesting parameters were the number of $-\mathrm{COOH}$ and $-\mathrm{PO}_{3} \mathrm{H}_{2}$ groups as well as the structure of the side chain in the amino acids. The experimental data proved that both the alkyl side chains (with or without additional carboxy and phosphonic groups) as well as the number of the ionizable substituents in the molecules influence the both efficacies.
\end{abstract}

Key words: inhibitor, corrosion, scale formation, $N$-carboxymethyl amino acid, $N$ phosphonomethyl amino acid.

Received: April 19, 2016. Published: April 26, 2016.

doi: $10.17675 / 2305-6894-2016-5-2-7$

\section{Introduction}

When both the corrosion and the scaling should be controlled in aqueous environment, it could happen via two procedures:

a. Two chemicals are simultaneously added to the system that can control the corrosion and the scaling [1]. The problem one has to face is that the parallel addition of the two different chemicals has an unwanted effect, i.e. their efficiency could be decreased by each other. The most frequently applied compositions consist of phosphonic acids and polymers with anions, carboxy and sulfo groups [2].

b. When one molecule can control the metal corrosion and the scale deposition they are mainly polymers: polycarboxylic acids (naphthalene polycarboxylic acid, acrylic polycarboxylic acids [3], itaconic acid-sodium allylsulfonate-sodium hypophosphite copolymer [4], or poly(aspartic acid - triptophane grafted polymer [5] as well as new phosphorous containing terpolymers [6]. Generally, the 
molecular weight of the polymers is between 200 and 5000. The efficiency of the inhibitors depends on the molecular weight, on the $\mathrm{pH}$, ion concentration, temperature and on the presence of other additives [7,8], which could be phosphonomethyl amine oxide [9], quaternary ammonium compounds [10] as well as aliphatic amino alcohols used together with diamino compounds [11].

The polymers that can efficiently control the scale formation are generally not biodegradable. This was the reason I have decided to work with small molecules with a natural basic part. In the case, when the chemicals from the treated industrial waters reach lakes and rivers, their biodegradation results in environmentally friendly molecules.

The other deteriorating process is the corrosion of metals. There are inhibitor molecules in wide range, their application depends on the environment they are used (solid surface, aqueous or oily liquids, temperature, pressure, etc.). Some of the most effective chemicals are those, which contain phosphono group(s). In these molecules, there is a covalent bond between the phosphorous and carbon atoms and this bond is very stable. Some examples are the hydroxyethane-diphosphonic acid, nitrilotri-phosphonic acid, 1phosphonobutane-tricarboxylic acid. Numerous papers describe their effectiveness [1219].

When we have decided to solve the inhibition of the above mentioned double unwanted processes, first we have synthesized N-carboxymethyl and N-phosphonomethyl substituted natural amino acids (their syntheses are not part of this paper) and then with special scale and gravimetric corrosion tests the influence of the molecule structures (ionic groups, alkyl chains) and effect of the $\mathrm{pH}$ were elucidated.

\section{Experimental}

\subsection{Materials}

Metal: Carbon steel was used in the corrosion experiments (composition: Fe 99.57\%, C $0.05 \%$, Si $0.05 \%$, Mn $0.3 \%$, S $0.01 \%$, P $0.02 \%$ ).

Inhibitors used in these experiments were as follows: N-carboxymethyl derivatives of glycine, alanine, $\beta$-alanine, norvaline, valine, aspartic acid, glutamic acid, phenylalanine, and lysine.

\subsection{Corrosion test}

The corrosion rates were determined by gravimetric technique and the efficiency $(\eta \%)$ calculated according to the equation:

$$
\eta=\frac{m_{0}-m_{i}}{m_{0}} \times 100[\%],
$$

where $m_{0}$ represents the weight loss of the coupon when inhibitor is absent, $m_{i}$ is the weight loss of the coupon measured in the presence of inhibitor. 
The corrosion tests were carried out in model solution $\left(\left[0.47 \mathrm{~g} \mathrm{CaSO}_{4} \times 2 \mathrm{H}_{2} \mathrm{O}+0.23 \mathrm{~g}\right.\right.$ $\left.\mathrm{MgSO}_{4} \times \mathrm{H}_{2} \mathrm{O}+0.11 \mathrm{~g} \mathrm{NaHCO}_{3}+0.13 \mathrm{~g} \mathrm{CaCl}_{2} \times \mathrm{H}_{2} \mathrm{O}\right] / 1 \mathrm{~L}$ distilled water, $\mathrm{pH}: 3.7$ and 8.5).

\subsection{Scale inhibition test}

$200 \mathrm{~mL}$ of the two model solutions $\left(3.12 \mathrm{~g} \mathrm{NaHCO}_{3} / 1 \mathrm{~L}\right.$ and $2.22 \mathrm{~g} \mathrm{CaCl}_{2} / 1 \mathrm{~L}$ in distilled water) were mixed, and after adding an aliquot of the $\mathrm{N}$-substituted amino acid solutions $(0.1 \%)$ the solution was heated to $60^{\circ} \mathrm{C}$. Samples taken after 2,4 , and 24 hours were filtered and the calcium content of the clear filtrate was determined by titration with ethylenediamine tetraacetic acid. The scale inhibiting activity was calculated by comparison of the calcium content in the original solution and in the samples with inhibitor, removed from the test solution.

\section{Results and discussion}

\subsection{Results of experiments measured in the presence of $N$-carboxymethyl amino acids}

As the Figure 1 demonstrates, this group of amino acid derivatives show antiscaling as well as anticorrosion activity, which depends on the side chain of amino acids. For the sake of comparison, the inhibitive activities of iminodiacetic acid and nitrilotriacetic acids (which have similar structures but are not derivatives of natural amino acids) are also involved in the figure. The efficiency data prove that most of the compounds studied can decrease the rate of corrosion and the scale deposition. Analyzing the structure-activity relationship, a branched side chain in the amino acid spoils the effect (see the case of valine $\rightarrow$ norvalin and $\beta$-alanine $\rightarrow$ alanine). The adverse effect could be explained by sterical hindrance. Longer alkyl chain is not favourale for scale inhibition, which is reflected in the antiscaling activity of alanine and norvaline derivatives. Data on anticorrosion effectiveness demonstrates the importance of the N-carboxymethyl aspartic acid. This activity is in the range of the efficacy of the nitrilotriacetic acid, which is an industrial scale deposition inhibitor. In the series of investigated $\mathrm{N}$-carboxymethyl amino acids, the substituted aspartic acid and glutamic acid are apt for industrial application.

\subsection{Results of experiments measured in the presence of of $N$-phosphonomethyl amino acids}

In the case of this group of compounds, not only the anticorrosion activity but the $\mathrm{pH}$ dependence of these data, too, was in the focus. As Figure 2 presents, the anticorrosive efficiency of these amino acid derivatives depends to a high degree upon the acidity of the test solution. 


\section{$\square$ scale inhibitors $\square$ corrosion inhibitors}

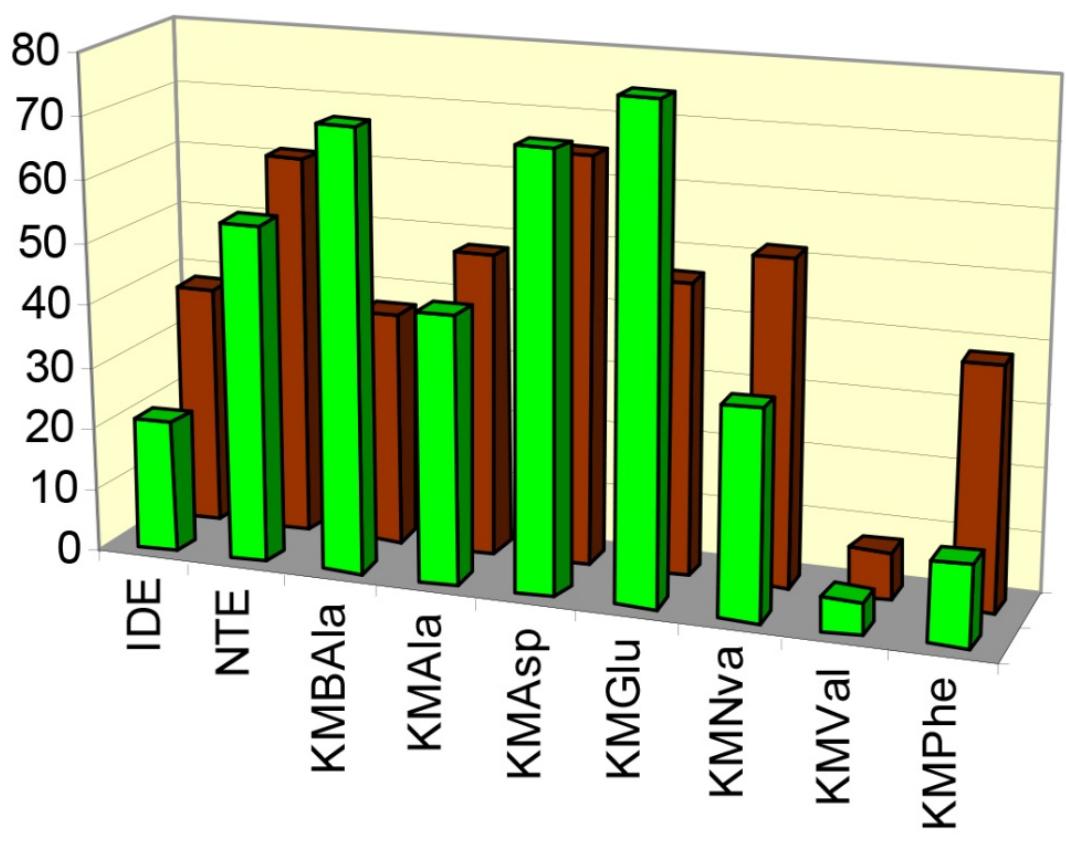

Figure 1. Carboxymethyl amino acids investigated in scale and corrosion tests; IDE: iminodiacetic acid, NTE: nitrilotriacetic acid; KM: carboxymethyl; BAla: $\beta$-alanine; Ala: alanine; Asp: aspartic acid; Glu: glutamic acid; NVal: norvalin; Val: valine; Phe: phenylalanine.

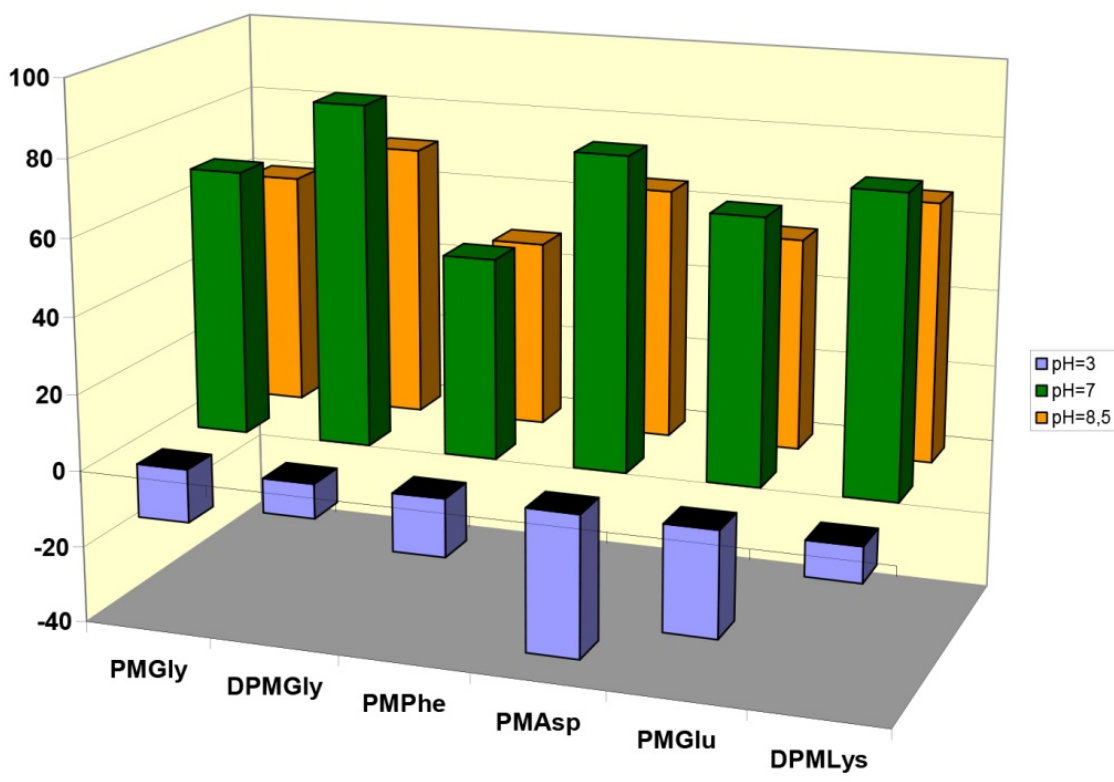

Figure 2. pH-dependent anticorrosion activities of $\mathrm{N}$-phosphonomethyl amino acids; PM: phospohonomethyl; DPM: diphosphonomethyl; Gly: glycine; Asp: aspartic acid; Glu: glutamic acid; Phe: phenylalanine; Lys: lysine. 
Numerical data are summarized in Table 1.

Table 1. The pH-dependent anticorrosion activities of N-phosphonomethyl amino acids.

\begin{tabular}{cccc}
\hline & $\begin{array}{c}\text { anticorr. } \\
\text { activity }\end{array}$ & $\begin{array}{c}\text { anticorr. } \\
\text { activity }\end{array}$ & $\begin{array}{c}\text { anticorr. } \\
\text { activity }\end{array}$ \\
N-phospohonomethyl amino acids & $\boldsymbol{\eta}[\%]$ & $\boldsymbol{\eta}[\%]$ & $\boldsymbol{\eta}[\%]$ \\
\cline { 2 - 4 } & $\mathbf{p H ~ 3}$ & $\mathbf{p H ~ 7}$ & $\mathbf{p H ~ 8 . 5}$ \\
\hline PMGly: $\mathrm{H}_{2} \mathrm{O}_{3} \mathrm{P}-\mathrm{CH}_{2}-\mathrm{NH}-\mathrm{CH}_{2}-\mathrm{COOH}$ & -14.1 & 69.3 & 60.7 \\
DPMGly: $\left(\mathrm{H}_{2} \mathrm{O}_{3} \mathrm{P}-\mathrm{CH}_{2}\right)_{2}-\mathrm{NH}-\mathrm{CH}_{2}-\mathrm{COOH}$ & -9.0 & 89.0 & 70.8 \\
PMPhe: $\mathrm{H}_{2} \mathrm{O}_{3} \mathrm{P}-\mathrm{CH}_{2}-\mathrm{NH}-\mathrm{CH}\left(\mathrm{CH}_{2}-\mathrm{C}_{6} \mathrm{H}_{5}\right)-\mathrm{COOH}$ & -15.1 & 52.1 & 48.3 \\
PMAsp: $\mathrm{H}_{2} \mathrm{O}_{3} \mathrm{P}-\mathrm{CH}_{2}-\mathrm{NH}-\mathrm{CH}\left(\mathrm{CH}_{2}-\mathrm{COOH}\right)-\mathrm{COOH}$ & -37.2 & 81.0 & 65.0 \\
PMGlu: $\mathrm{H}_{2} \mathrm{O}_{3} \mathrm{P}-\mathrm{CH}_{2}-\mathrm{NH}-\mathrm{CH}\left(\mathrm{CH}_{2}-\mathrm{CH}_{2} \mathrm{COOH}\right)-\mathrm{COOH}$ & -27.5 & 68.4 & 45.0 \\
DPMLys: $\mathrm{H}_{2} \mathrm{O}_{3} \mathrm{P}-\mathrm{CH}_{2}-\mathrm{NH}-\mathrm{CH}\left(\left(\mathrm{CH}_{2}\right)_{4}-\mathrm{NH}-\mathrm{CH}-\mathrm{H}_{2} \mathrm{O}_{3} \mathrm{P}\right)-\mathrm{COOH}$ & -9.1 & 77.1 & 67.2 \\
\hline
\end{tabular}

The presence of more ionizable groups in the amino acid side chain increases significantly the anticorrosion efficiency. When an inhibitive layer is formed on the metal surface, the carboxy and phosphono groups face the oxide layer covered metal surface and form a physical bond. When the number of these groups increases, the coverage of the surface with inhibitor molecules increases too; the aggressive components of the environment/solution cannot come near to the metal surface. The consequence is that the rate of corrosion decreases. The $\mathrm{pH}$-dependence of the efficacy unequivocally proved that these molecules are not proper inhibitors for example in etching liquids as all of the investigated molecules do not decease but increase the metal dissolution under acidic condition. It shows that these molecules in protonated form cannot inhibit properly the metal corrosion. On the other hand, when the environment is neutral or mildly alkali, these additives decrease the rate of corrosion significantly and they are good inhibitors. When the molecular structure is under investigation, data demonstrate that the efficacy decreases when a phenyl ring is present in the side chain of the amino acid, as the case of the phenylalanine derivative shows. The most effective additives are the N,N-disubstituted amino acid, the activity does not depend on the length of the alkyl chain in the amino acid.

Not only the anticorrosion property of these molecules was followed, but their inhibition of scale deposition was also measured. In order to compare these activities, Figure 3 summarizes their antiscaling and anticorrosion effectiveness.

The same molecule i.e. $\mathrm{N}, \mathrm{N}$-disubstituted glycine was the most effective inhibitor of scale deposition as well as of corrosion. 


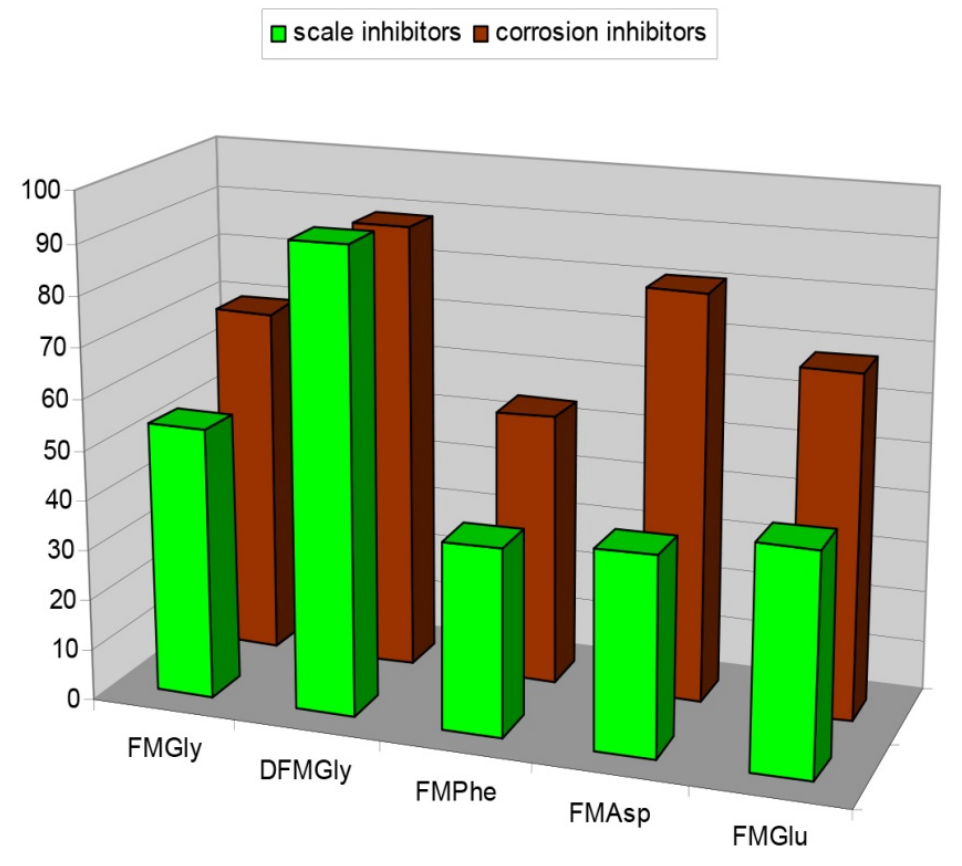

Figure 3. N-phosphonomethyl amino acids as inhibitors of scale formation and corrosion; PM: phospohonomethyl; DPM: diphosphonomethyl; Gly: glycine; Asp: aspartic acid; Glu: glutamic acid; Phe: phenylalanine.

\section{Conclusion}

Summarizing results got in the presence on N-carboxymethyl and N-phosphonomethyl amino acids it unravels that the increase in number of phosphono groups in the substituted amino acids calls forth significant increase in effectiveness of both activities. The impact of the alkyl side chain in the amino acid is less important than the presence of additional carboxylic groups when already phosphono group is present in a molecule; this is explainable by the chelate formation at the metal/metal oxide surface via carboxylic and phosphono groups. This enlightens the increase in the efficiency on increase in the number of functional groups.

The $\mathrm{pH}$-dependent corrosion rates reveal on the importance of the molecular state. Comparing the negative effect of the acidic medium on the corrosion rate with those data got at neutral or slightly alkali $\mathrm{pH}$, it is clear that in the adsorption processes at the metal/metal oxide interface mainly those molecules take part where the carboxylic and phosphonic groups are not protonated.

From this group of compounds the N,N-di(phosphonomethyl)glycine can most effectively control both the corrosion and the scale deposition. The systematic survey confirms unequivocally that the equivalent number of the carboxylic groups does not show the same increase in effectiveness than that of the phosphono analogs. 


\section{References}

1. A. Al-Borno, M. Islam and R. Khraishi, Corrosion, 1989, 42, 970.

2. F. Moran, 1986, PCT Int. Appl. WO 9604411 A1.

3. Ch. Carber, G. Kreh, R.P. Fan and L. Duinen, 1992, US 5139702 A1.

4. Zh. Liu, H. Fu and L. Zhang, Desalination, 2016, 57, 5740.

5. X. Sun, J. Zhang, Ch. Yin, J. Zhang and J. Han, J. Appl. Polym. Sci., 2015, 132, 42739. doi: 10.1002/app.42739

6. Y. Chen, Y. Zhou, Q. Yao, Y. Bu and H. Wang, Desalin. Water Treat., 2015, 55, 945.

7. B. Clubley and G. Riddent, 1993, J. EP 544345 A1.

8. J.L. Fang, Y. Li and X.R. Ye, Corrosion (Houston, TX, U.S.), 1993, 49, 266.

9. Ch.M. Hwa, J.A. Kelly, J. Neton, P.M. Scanlon and R.R. Gaudette, 1994, EP 437722 B1.

10. A.J. Swift, Microchim. Acta, 1995, 120, 149.

11. M. Duprat and F. Dabosi, Corrosion (Houston, TX, U.S.), 1981, 37, 89.

12. J. Telegdi, E. Kálmán and F.H. Kármán, Corros. Sci., 1992, 33, 1099.

13. J. Telegdi, $A C H$ - Models Chem., 1995, 132, 741.

14. Yu.I. Kuznetsov, Progress in the Understanding and Prevention of Corrosion, in The Phosphonate inhibitors of Metal Corrosion, Wiley-VCH, 1995, pp. 853-859.

15. E. Kálmán, I. Felhősi, F.H. Kármán, I. Lukovits, J. Telegdi and G. Pálinkás, Materials Science and Technology; A Comprehensive Treatment, Corrosion and Environmental Degradation, Eds. R.W. Chan, P. Haasen and E.J. Kramer, 2000, Vol. 1, pp. 472-537, Wiley-VCH.

16. D.-J. Choi, S.-J. You and J.-G. Kim, Mater. Sci. Eng., A, 2002, 335, 228.

17. H. Amar, J. Benzakour, A. Derja, D. Villemin, B. Moreau and T. Braisaz, Appl. Surf. Sci., 2006, 252, 6162.

18. M. Prabakaran, M. Venkatesh, S. Ramesh and V. Periasamy, Appl. Surf. Sci., 2013, 276, 592.

19. S. S. Rao, B.V. Appa Rao, S. Roopas Kiran and B. Sreedhar, J. Mater. Sci. Technol., 2014, 30, 77. 